Estudo de Caso / Breve Relato

Case Study / Brief Report

\title{
Efeito agudo da ventilação não invasiva associada ao exercício físico sobre parâmetros cardiovasculares e respiratórios: um estudo de caso
}

\section{Acute Effect of Non-Invasive Ventilation Associated with Physical Exercise on Cardiovascular and Respiratory Parameters: a Case Study}

\author{
Guilherme Rosa ${ }^{\S 1,2} \mathrm{PhD}$; Tais Paes Guedes ${ }^{1}$; Thiago de Andrade Silva ${ }^{1}$; Fábio Dutra ${ }^{1,3} \mathrm{PhD}$ \\ Recebido em: 07 de março de 2018. Aceito em: 21 de abril de 2018 \\ Publicado online em: 11 de julho de 2018.
}

\section{Resumo}

Introdução: 0 método de suprimento de ventilação não invasivo (VNI) durante a realização de exercícios físicos tem sido utilizado como uma ferramenta auxiliar no tratamento de pacientes com doenças pulmonares. A literatura mostra que ainda há escassez de dados acerca dos efeitos da utilização da VNI durante a realização do exercício físico sobre os parâmetros cardiorrespiratórios.

Objetivo: Avaliar o efeito do exercício físico associado a VNI sobre parâmetros cardiovasculares e respiratórios.

Métodos: Participou deste estudo de caso um indivíduo do sexo masculino, saudável, com 27 anos de idade. Foram mensurados: frequência cardíaca (FC), variabilidade cardíaca (VFC), saturação periférica de oxigênio $\left(\mathrm{SPO}_{2}\right)$, pressão arterial sistólica (PAS) e diastólica (PAD) e duplo produto (DP). Foram realizados dois treinos cardiorrespiratórios em esteira ergométrica, um sem VNI e o outro com VNI. As sessões de treinamento contemplaram duração de 40 min divididos em 5 min de aquecimento com intensidade entre $50 \%$ e $55 \%$ da FC reserva, 30 min de fase específica com intensidade entre $60 \%$ e $85 \%$ da FC reserva, e 5 min de desaquecimento com intensidade de $50 \%$ a $55 \%$ da FC reserva. Para avaliar as alterações das variáveis desfecho, foi estimada a variação percentual pelo coeficiente de variação.

Resultados: A realização do exercício com VNI provocou alterações nos parâmetros cardiovasculares e respiratórios analisados, com destaque para redução da $\operatorname{VFC}(\Delta \%=-64,14)$ e $\operatorname{PAD}(\Delta \%=-45,82)$ em comparação à realização sem VNI.

Conclusão: Os resultados do presente estudo sugerem que a realização do exercício físico com VNI pode beneficiar as respostas dos parâmetros cardiovasculares e respiratórios. Os resultados foram discutidos.

Palavras-chave: exercício físico, sistema respiratório, pressão positiva contínua nas vias aéreas.

\begin{abstract}
Introduction: The noninvasive ventilation (NIV) method during physical exercise has been used as an auxiliary tool in the treatment of patients with pulmonary diseases. However, there is a lacune on the knowledge about cardiorespiratory parameters effects of NIV during physical exercise.

Objective: To evaluate the effect of physical exercise associated with NIV on cardiovascular and respiratory parameters.
\end{abstract}

\footnotetext{
${ }^{\S}$ Autor correspondente: Guilherme Rosa-grfitness@hotmail.com Afiliações: ${ }^{1}$ Universidade Castelo Branco - UCB/RJ; ${ }^{2}$ Grupo de Pesquisas em Exercício Físico e Promoção da Saúde - UCB/RJ; 3/nFocus Research Group - UCB/RJ.
} 
Methods: A healthy, 27-year-old male was enrolled in this case study. Heart rate (HR), cardiac variability (HRV), peripheral oxygen saturation $\left(\mathrm{SPO}_{2}\right)$, systolic blood pressure (SBP) and diastolic blood pressure (DBP) and double product (SD) were measured. Two cardiorespiratory treadmills were performed on treadmill, one without NIV and the other with NIV. The training sessions included 40 min duration divided in 5 min of heating with intensity between $50 \%$ and $55 \%$ of reserve HR, 30 min of specific phase with intensity between $60 \%$ and $85 \%$ of reserve HR, and 5 min of cooling with intensity of $50 \%$ to $55 \%$ of reserve FC. To express the changes of the variables, the percentage change was estimated by the coefficient of variation.

Results: The exercise with NIV caused changes in cardiovascular and respiratory parameters, with a reduction in $\operatorname{HRV}(\Delta \%=-64.14)$ and DBP $(\Delta \%=-45.82)$ in comparison with non-NIV.

Conclusion: Our findings suggested that physical exercise with NIV use may benefit in association with exercise in relation to cardiovascular and respiratory parameters. The results were discussed.

Keywords: exercise, respiratory system, continuous positive airway pressure.

\section{Efeito agudo da ventilação não invasiva associada ao exercício físico sobre parâmetros cardiovasculares e respiratórios: um estudo de caso}

\section{Introdução}

Doenças pulmonares têm como principais características a diminuição da capacidade respiratória, decorrentes da inflamação do pulmão por intolerância a partículas e/ou gases nocivos(1). Tais condições podem levar o paciente a limitação ventilatória, disfunção na oferta e no consumo de oxigênio, inflamação sistêmica, estresse oxidativo e débito cardíaco alterado. Associado a isso, está o quadro de hipoxemia pré exercício, que pode levar esses indivíduos a dispneias quando submetidos a atividades físicas(2), produzindo a cessação do esforço a baixas cargas de trabalho, o que induz ao descondicionamento progressivo, anulando, assim, a performance dos músculos periféricos e respiratórios desses pacientes(3).

A literatura mostra que a baixa capacidade respiratória em pacientes com doenças pulmonares pode ser aprimorada discretamente por terapias clínicas(4) e a aplicação da terapia de exercício físico tem sido utilizada como tratamento coadjuvante(5). Isto se explica porque o exercício físico se apresenta como ferramenta

complementar para a melhora da resistência aeróbica, que tem papel importante na redução da demanda respiratória e na sensação de dispneia(4). A presença de tal sensação, faz

\section{Keypoints}

- Physical exercise with NIV induced changes in HRV ( $p=$ $0.03)$ and $P D(p=0.0001)$.

- There were no changes for $F C$, $S P O_{2}, P A S$ and $P A D$.

- There was a positve trend in the use of NIV in association with physical exercise. 
oxigênio em repouso apresentem também baixa capacidade a exercícios aeróbicos, quando submetidos a teste de caminhada de 6 minutos(2).

Uma das doenças pulmonares mais frequentes é a doença pulmonar obstrutiva crônica (DPOC). Tradicionalmente, o agravamento da DPOC é definido pelo grau de obstrução das vias aéreas, que é avaliada pelo volume expiratório forçado em um segundo, pela capacidade inspiratória e pela capacidade pulmonar total $(7,8)$.

O método de suprimento de ventilação não invasivo (VNI) tem apresentado resultados valiosos, quando usado como uma ferramenta auxiliar em indivíduos com doenças pulmonares contribuindo para promover melhora na função respiratória e o aumento da tolerância ao exercício físico(9). A VNI favorece a compensação dos índices de função pulmonar e muscular respiratória, resultando em benefícios como aumento da resistência à fadiga muscular e diminuição da sensação de desconforto nos membros inferiores em altas cargas de treinamento(10). Entretanto, ainda há escassez de estudos acerca da utilização da VNI durante a realização do exercício físico sobre parâmetros cardiovasculares e respiratórios.

Portanto, o objetivo do presente experimento foi avaliar o efeito agudo do exercício físico associado a VNI sobre parâmetros cardiovasculares e respiratórios em um indivíduo saudável.

\section{Métodos}

\section{Desenho de estudo e amostra}

A presente investigação, do tipo experimental, caracteriza-se como um estudo de caso do tipo avaliativo, descritivo, pois, trata-se de uma forma de pesquisa em que um único caso é estudado em profundidade na tentativa de alcançar uma compreensão maior sobre casos semelhantes $(11,12)$. O caso foi composto por um indivíduo do sexo masculino, de 27 anos de idade, praticante regular de exercícios físicos, sem uso de medicamentos que pudessem alterar as variáveis dependentes do estudo, bem como seu desempenho no teste, e sem fator de risco aparente de acordo com os critérios de estratificação de risco da American Heart Association - AHA(13).

\section{Aspectos éticos}

Os procedimentos experimentais foram executados de acordo com as normas éticas previstas na Resolução 466/2012 do Conselho Nacional de Saúde quanto à pesquisa científica envolvendo seres humanos. O participante assinou o termo de consentimento livre e esclarecido às medições a que foi submetido.

\section{Variáveis de estudo}

As variáveis hemodinâmicas e cardiorrespiratórias: frequência cardíaca (FC); variabilidade da frequência cardíaca (VFC); saturação parcial de oxigênio $\left(\mathrm{SPO}_{2}\right)$; pressão arterial sistólica (PAS); pressão arterial diastólica (PAD); e duplo produto (DP) foram as variáveis desfecho.

A variável de exposição foi a VNI, durante a realização de exercício físico.

Medidas antropométricas, estado nutricional (Índice de Massa Corporal: IMC), cálculo do percentual de gordura foram as covariáveis, utilizadas para descrever a amostra.

\section{Medidas antropométricas}

Foram aferidas as medidas de massa corporal (MC) e estatura (EST) através de uma balança mecânica com estadiômetro acoplado da marca Welmy®, com precisão de $100 \mathrm{~g}$ e capacidade de $150 \mathrm{~kg}$. Com base nos resultados, foram calculados o índice de massa corporal (IMC) por meio da razão entre a MC e o quadrado da EST.

O percentual de gordura corporal $(\% \mathrm{G})$ foi estimado pela da equação de Siri e do protocolo de Jackson; Pollock(14) para estimativa da densidade corporal por 3 dobras cutâneas utilizando o compasso da marca Sanny ${ }^{\circledR}$. As medidas antropométricas seguiram os procedimentos recomendados pela International Society for Advance of Kinanthropometry - ISAK(15).

\section{Procedimentos experimentais}

A intervenção do estudo foi treino cardiorrespiratório na esteira ergométrica por meio do cálculo da zona alvo de treinamento, considerando a frequência cardíaca de reserva. $\mathrm{O}$ controle da intensidade foi feito pelo frequencímetro Polar ${ }^{\circledR}$ modelo RS800, no entanto concomitante utilizou-se o oxímetro de 
dedo ChoiceMMed® modelo MD300C1 Fingertip garantindo a saturação acima de $90 \%$, a pressão arterial sistólica e diastólica foram aferidas usando o monitor de pressão arterial automático Omron ${ }^{\circledR}$ modelo HEM7113. Os dados foram coletados quando em repouso a cada 5 minutos e quando em exercício a cada 10 minutos.

As sessões de treinamento contemplaram um volume de 40 minutos divididos em 5 minutos de aquecimento com intensidade de $50 \%$ a $55 \%, \quad 30$ minutos de treinamento cardiorrespiratório através do método contínuo com intensidade entre $60 \%$ e $85 \%$ e finalizando 5 minutos de desaquecimento com intensidade de $50 \%$ a $55 \%(15,16)$.

A intervenção se deu durante duas semanas de treino, sendo uma sessão por semana. No primeiro dia, o participante fez a sessão de treinamento sem o auxílio da VNI. No segundo dia, o participante realizou o exercício com VNI, utilizando o equipamento Continuous Positive Airway Pressure (CPAP) Respironics ${ }^{\circledR}$ durante o exercício. A máscara foi colocada no participante 10 minutos antes de iniciar o treinamento, para a adaptação à pressão da mesma, e retirada 10 minutos após o término da sessão de treinamento. $O$ nível médio de pressão positiva inspiratória aplicada nas vias aéreas foi de $10 \mathrm{cmH}_{2} \mathrm{O}$.

$\mathrm{O}$ participante foi orientado a realizar a micção antes do início da intervenção. Foi permitido ao longo da prática a ingestão de 250 $\mathrm{ml}$ de água. A temperatura ambiente do laboratório foi de $21^{\circ} \mathrm{em}$ ambas as sessões de treinamento.

\section{Análise estatística}

Foi realizada a análise descritiva dos dados através de medidas de tendência central e de dispersão (média \pm desvio padrão). Para avaliar as alterações das variáveis nos distintos momentos do estudo, foi estimada a variação percentual utilizando-se o coeficiente de variação.

\section{Resultados}

O caso foi composto por um participante de 27 anos, cujas características descritivas apresentam-se na Tabela 1.
Tabela 1 - Características antropométricas e de composição corporal do voluntário

\begin{tabular}{ccccc}
\hline Idade & MC (kg) & Est (m) & IMC & $\% \boldsymbol{G}$ \\
\hline \hline 27,00 & 78,69 & 1,77 & 27,66 & 22,93 \\
\hline
\end{tabular}

MC: massa corporal total; Est: estatura; IMC: índice de massa corporal; \%G: percentual de gordura corporal.

Durante a coleta de dados, na segunda sessão de treinamento, o realizado com VNI utilizando a máscara facial, por apresentar maior conforto quando comparada a nasal.

Os valores das variáveis coletadas com e sem a utilização da VNI nos momentos repouso, exercício e recuperação estão expostas na Tabela 2.

Com base nos dados apresentados na tabela 2, é possível observar que a VNI provocou elevação da $\mathrm{FC}$ e redução $\mathrm{VFC}$ nos distintos momentos que compuseram o estudo. Para a PAS, a VNI induziu redução durante o repouso e o exercício, com aumento durante a recuperação. $\mathrm{A} \quad \mathrm{SPO}_{2}$ não apresentou alterações durante o repouso em resposta à VNI, entretanto, durante o exercício e a recuperação, foram observadas reduções da mesma magnitude. Durante o exercício, a VNI foi capaz de provocar redução nos valores da PAD, diferentemente do observado no repouso e na recuperação, onde houve aumento. Quanto ao DP, houve redução da variável com a utilização da VNI nos momentos repouso e exercício, com aumento durante a recuperação.

\section{Discussão}

Com o objetivo de investigar os efeitos do suporte ventilatório não invasivo, por meio do CPAP, sobre o comportamento das variáveis fisiológicas $\mathrm{FC}, \mathrm{VFC}, \mathrm{SPO}_{2}, \mathrm{PAS}, \mathrm{PAD}$ e $\mathrm{DP}$, foi possível observar algumas alterações.

Ao se analisar a variável FC, o presente estudo demonstrou elevação dos níveis das variáveis desfecho em resposta à utilização da VNI, independentemente do momento analisado. Tais achados divergem em relação aos resultados de Pissulin et al.(17), que apontaram redução significativa da variável $\mathrm{FC}$ em reposta ao uso da VNI durante exercício também realizado em esteira ergométrica. Este fato pode ter ocorrido por conta da diferença entre o fluxo com o qual o CPAP fora regulado nos estudos. 
Tabela 2 - Efeito agudo da realização de exercício sem e com ventilação não invasiva (VNI)

\begin{tabular}{lccc}
\hline Variáveis/Momentos & $\begin{array}{c}\text { SEM VNI } \\
\boldsymbol{\mu} \pm \text { DesvP }\end{array}$ & $\begin{array}{c}\text { COM VNI } \\
\boldsymbol{\mu} \pm \text { DesvP }\end{array}$ & $\boldsymbol{\Delta} \%$ \\
\hline \hline Repouso & & & \\
FC rep & $77,0 \pm 1,4$ & $83,0 \pm 0$ & 7,79 \\
VFC rep & $30,8 \pm 3,5$ & $17,5 \pm 2,4$ & $-43,18$ \\
SPO rep & $99,0 \pm 0$ & $99,0 \pm 0$ & 0,00 \\
PAS rep & $143,5 \pm 6,3$ & $131,0 \pm 4,2$ & $-8,71$ \\
PAD rep & $80,5 \pm 0,7$ & $82,0 \pm 1,4$ & 1,86 \\
DP rep & $11045 \pm 287,0$ & $10873,0 \pm 352,0$ & $-1,56$ \\
Exercício & & & \\
FC ex & $158,8 \pm 9,8$ & $165,2 \pm 12,1$ & 4,03 \\
VFC ex & $10,04 \pm 4,2$ & $3,6 \pm 0,8$ & $-64,14$ \\
SPO ex & $97,6 \pm 1,1$ & $96,6 \pm 1,3$ & $-1,02$ \\
PAS ex & $139,2 \pm 8,4$ & $134,0 \pm 15,4$ & $-3,74$ \\
PAD ex & $134,0 \pm 10,0$ & $72,6 \pm 3,0$ & $-45,82$ \\
DP ex & $22043,0 \pm 543,0$ & $22020,0 \pm 1596,0$ & $-0,10$ \\
Recuperação & & & \\
FC rec & $96,0 \pm 2,8$ & $102,0 \pm 2,8$ & 6,25 \\
VFC rec & $17,7 \pm 8,4$ & $9,1 \pm 0,3$ & $-48,59$ \\
SPO rec & $98,5 \pm 0,7$ & $97,5 \pm 0,7$ & $-1,02$ \\
PAS rec & $113 \pm 2,8$ & $114,5 \pm 2,1$ & 1,33 \\
PAD rec & $67,5 \pm 0,7$ & $76,0 \pm 1,4$ & 12,59 \\
DP rec & $10842,0 \pm 48,0$ & $11676,0 \pm 107,0$ & 7,69 \\
\hline
\end{tabular}

$\mu \pm$ DesvP : média \pm desvio padrão; $\Delta \%$ : variação percentual resultado do cálculo do coeficiente de variação entre os momentos SEM VNI e COM VNI; rep: repouso; ex: exercício; rec: recuperação; FC: frequência cardíaca; VFC: variabilidade da frequência cardíaca; $\mathbf{S P O}_{2}$ : saturação parcial de oxigênio; PAS: pressão arterial sistólica; PAD: pressão arterial diastólica; DP: duplo produto.

A VFC apresentou resultado relevante. O treinamento com uso da VNI foi capaz de promover melhora no domínio e na modulação do efeito parassimpático da FC, como ocorreu também no estudo de Skyba et al.(18), que usou pressão positiva nas vias aéreas no treinamento aeróbico de indivíduos com DPOC.

Os resultados exibidos em PAS e PAD, demonstraram que a utilização da VNI produziu efeitos similares durante o exercício e a recuperação, com diferença nas respostas durante o repouso. Além disso, observa-se que a utilização da VNI durante o exercício gerou respostas da PAS mais baixas em comparação com o exercício sem VNI. O estudo realizado por Skyba et al.(18) também demonstrou resultados favoráveis, similares aos observados na presente investigação, com redução significativa da PAS durante $o$ exercício com a utilização da VNI, em pessoas com DPOC. Normalmente, tais pacientes tendem a apresentar, em repouso, hipoxemia e hipercapnia, decorrente da dificuldade de realizar hematose pulmonar, assim acarretando baixos níveis de saturação parcial de oxigênio sanguíneo $\left(\mathrm{SPO}_{2}\right)(19)$. O uso da VNI em pacientes com esse quadro clínico, tem contribuído para melhoras significativas nesse parâmetro, como aponta o estudo de Dreher et al.(20). o presente trabalho não mostrou resultados relevantes para essa variável, com nenhuma alteração durante $\mathrm{o}$ repouso, e discretas modificações durante o exercício e a recuperação, sem sair, entretanto, da zona de normalidade $(95 \%)$. Tal fato provavelmente está ligado ao indivíduo ser saudável e possivelmente não apresentar nenhuma deficiência pulmonar.

Com relação à variável $\mathrm{DP}$, o presente estudo apresentou uma discreta redução observada entre a fase de repouso e a sessão de

\footnotetext{
§Autor correspondente: Guilherme Rosa-grfitness@hotmail.com Afiliações: ${ }^{1}$ Universidade Castelo Branco - UCB/RJ; ${ }^{2}$ Grupo de Pesquisas em Exercício Físico e Promoção da Saúde - UCB/RJ; ${ }^{3}$ InFocus Research Group - UCB/RJ.
} 
exercício, bem como o ligeiro aumento durante o período de recuperação. Esse efeito pode estar associado à pressão que o CPAP é capaz de produzir nas vias aéreas, diminuindo assim a hiperinsuflação dos pulmões e melhorando a sua mecânica respiratória deixando o indivíduo mais confortável em exercício(17).

Com relação a variável $\mathrm{DP}$, o presente estudo apresentou uma discreta redução observada durante o repouso e a sessão de exercício, bem como o ligeiro aumento durante o período de recuperação. Esse efeito pode estar associado a pressão que o CPAP é capaz de produzir nas vias aéreas, diminuindo assim a hiperinsuflação dos pulmões e melhorando a sua mecânica respiratória deixando o indivíduo mais confortável em exercício(17).

Os resultados observados no presente estudo sugerem que, para melhor compreender os mecanismos que promovem a melhora de parâmetros cardiorrespiratórios em pacientes com doenças pulmonares, novos estudos devem incluir tanto pacientes quanto pessoas saudáveis em suas amostras.

\section{Pontos fortes e limitações do estudo}

Um ponto forte do estudo foi a investigação a respeito do efeito agudo do exercício realizado com VNI, nas variáveis cardiorrespiratórias, em indivíduo saudável. Os resultados contribuem para uma maior compreensão dos mecanismos que favorecem a utilização desse método no tratamento coadjuvante para pacientes com doenças pulmonares. A relevância do estudo fica destacada por não ter sido identificado nenhum estudo que tivesse investigado a realização com e sem VNI em pessoas saudáveis.

As limitações do presente estudo residem principalmente no tamanho amostral, motivo pelo qual não se aplicou análise estatística quanto à significância das diferenças observadas.

\section{Conclusão}

O objetivo do estudo foi avaliar o efeito agudo do exercício físico associado a VNI sobre parâmetros cardiovasculares e respiratórios em um indivíduo saudável, para contribuir com a segurança da aplicação da VNI durante a realização de exercício em pacientes com doenças pulmonares.
Observaram-se alterações positivas tendência em relação à utilização da VNI, durante o exercício físico, em relação as respostas dos parâmetros cardiovasculares e respiratórios avaliados.

Os achados foram positivos, entretanto, face às limitações do estudo, sugere-se que sejam conduzidas novas investigações, que contemplem a temática, modificando-se as características do exercício, utilizando uma amostra maior e composta tanto por pacientes com doenças pulmonares quanto por pessoas saudáveis para que possíveis diferenças sejam identificadas na comparação de ambos e seja possível maior compreensão dos mecanismos envolvidos na melhora dos parâmetros cardiorrespiratórios desses pacientes.

\section{Declaração de conflito de interesses}

Não há nenhum conflito de interesses no presente estudo.

\section{Declaração de financiamento}

O presente estudo foi desenvolvido sem financiamento.

\section{Referências}

1. Silva KR, Marrara KT, Marino DM, Di Lorenzo VAP, Jamami M. Fraqueza muscular esquelética e intolerância ao exercício em pacientes com doença pulmonar obstrutiva crônica. Revista Brasileira de Fisioterapia. 2008;12(3):169-175.

2. Brunetto AF, Pitta FO, Probst VS, Paulin E, Yamaguti WPS, Ferreira LF. Influência da saturação de $\mathrm{O} 2$ na velocidade do teste de distância percorrida em 6 minutos, em pacientes com DPOC grave. Revista Brasileira de Fisioterapia. 2003;7(2):123129.

3. Borghi AS, Sampaio LMM, Toledo A, Pincelli MP, Costa D. Efeitos agudos da aplicação do BIPAP sobre a tolerância ao exercício físico em pacientes com doença pulmonar obstrutiva crônica (DPOC). Revista Brasileira de Fisioterapia. 2005;9(3):273-280.

4. Dourado VZ, Godoy I. Recondicionamento muscular na DPOC: principais intervenções e novas 
tendências. Revista Brasilera de Medicina do Esporte. 2004;10(4):331-334.

5. Hill K, Holland AE. Strategies to Enhance the Benefits of Exercise Training in the Respiratory Patient. Clinics In Chest Medicine. 2014; 35(2): 323-336.

6. Paulin E, Brunetto AF, Carvalho CRF. Efeitos de programa de exercícios físicos direcionados ao aumento da mobilidade torácica em pacientes portadores de doença pulmonar obstrutiva crônica. Journal of Pneumology. 2003;29(5): 287294.

7. Freitas CG, Pereira CAC, Viegas CAA. Capacidade inspiratória, limitação ao exercício, e preditores de gravidade e prognóstico, em doença pulmonar obstrutiva crônica. Jornal Brasileiro de Pneumologia. 2007;33(4):389-396.

8. Vilaró J, Resqueti VR, Fregonezi GAF. Avaliação clínica da capacidade do exercício em pacientes com doença pulmonar obstrutiva crônica. Revista Brasileira de Fisioterapia. 2008;12(4):249-259.

9. Ambrosino N, Strambi S. New strategies to improve exercise tolerance in chronic obstructive pulmonary disease. European Respiratory Journal. 2004; 24(2): 313322.

10. Moreno J, Corso SD, Malaguti C. Análise descritiva do uso de ventilação mecânica não invasiva durante exercício em pacientes com DPOC. ConScientiae Saúde. 2007;6(2):295-303.

11. Gil A C. Como elaborar projetos de pesquisa. $4^{\mathrm{a}}$ ed. São Paulo: Atlas; 2007.

12. Alves Mazzotti AJ, Gewandsznajder F. $O$ método nas ciências naturais e sociais: pesquisa quantitativa e qualitativa. São Paulo: Pioneira; 1998.

13. ACSM. Manual do ACSM para avaliação da aptidão física relacionada à saúde. 3 Ed: Guanabara Koogan; 2011.

14. Marfell-Jones T, Stewart A, Carter L. International standards for anthropometric assessment. ISAK:
International Society for the Advancement of Kinanthropometry,South Africa. 2006.

15. Silva AB, Mendes RG, Trimer R, Oliveira CR, Fregonezi GAF, Resqueti VR, et all. Potential effect of 6 versus 12-weeks of physical training on cardiac autonomic function and exercise capacity in chronic obstructive pulmonary disease. European Journal of Physical and Rehabilitation Medicine. 2015;51(2):211-221.

16. Dourado VZ, Tanni SE, Antunes LCO, Paiva SAR, Campana AO, Renno ACM, at all. Effect of three exercise programs on patients with chronic obstructive pulmonary disease. Brazilian Journal of Medical and Biological Research. 2009;42(3):263-271.

17. Pissulin FDM, Guimarães A, Kroll LBU, Cecílio MJ. Utilização da pressão positiva contínua nas vias aéreas (CPAP) durante atividade física em esteira ergométrica em portadores de doença pulmonar obstrutiva crônica (DPOC): Comparação com o uso de oxigênio. Jornal Brasileiro de Pneumologia. 2002; 28(3): 131-136.

18. Skyba P, Joppa P, Orolín M, Tkáčová R. Blood pressure and heart rate variability response to noninvasive ventilation in patients with Exacerbations of Chronic Obstructive Pulmonary Disease. Physiological Research. 2007;56: 527533.

19. Santana VTS, Squassoni SD, Neder JA, Fiss E. Influência do tabagismo atual na aderência e nas respostas à reabilitação pulmonar em pacientes com DPOC. Revista Brasileira de Fisioterapia. 2010;14(1):16-23.

20. Dreher M, Storre JH, Windisch W. Noninvasive ventilation during walking in patients with severe COPD: a randomized cross-over trial. European Respiratory Journal. 2007; 29(5):930-936. 